\title{
RETRACTION: The Impact of Authenticated Epistemologies on Hardware and Architecture
}

\author{
Bing $\mathrm{Li}^{*}$, Lianyong Zhou and Li Chen \\ Beijing Zhongdianhuayuan Technology Company, Beijing, 100011, China
}

In April 2020, Atlantis Press was alerted to fake proceedings articles hosted on our platform. These articles, mostly in computer science and engineering, were generated by the SCIgen computer program and were all published between 2006-2018. Immediately after receiving the alert, Atlantis Press initiated a thorough investigation and contacted the relevant conference organizers and authors. As a member of the Committee on Publication Ethics (COPE), we followed COPE-compliant guidelines and procedures while investigating these cases. Based on this, it was concluded that all these proceedings articles should be retracted. This article (https://doi.org/10.2991/emcm-15.2016.65) was one of the affected cases and has therefore been retracted accordingly. Since 2018, strong editorial and publishing control measures were put in place to reject fake submissions, unethical research and low-quality papers. We also conduct regular audits of our existing procedures to identify improvements to processes and to avoid such issues from happening again.

The Publisher apologizes for any inconvenience this may cause.

The full Atlantis Press Policy on Article Retraction can be found at: https://www.atlantispress.com/policies/article-retraction-and-withdrawal 